\title{
Collaborative Energy Conservation in a Microgrid
}

\author{
Mohit Jain $^{I}$, Harshad Khadilkar $^{I}$, Neha Sengupta ${ }^{I *}$, Zainul Charbiwala ${ }^{I}$, \\ Kushan $U$ Tennakoon $^{\mathcal{B}}$, Rodzay bin Haji Abdul Wahab ${ }^{\mathcal{B}}$, \\ Liyanage Chandratilak De Silva ${ }^{\mathcal{U}}$, Deva P. Seetharam ${ }^{I *}$ \\ ${ }^{I}$ IBM Research, ${ }^{\mathcal{B}}$ Institute for Biodiversity \& Environmental Research, \\ UUniversiti Brunei Darussalam \\ \{mohitjain,harshad.khadilkar,zainulcharbiwala\}@in.ibm.com
}

\begin{abstract}
KBFSC (Kuala Belalong Field Studies Centre) is a research centre located in a remote tropical evergreen rainforest in Brunei Darussalam in South East Asia. It is visited by biologists and ecologists from all over the world. Power is available at the centre for 8-10 hours per day from a diesel generator (DG). The diesel travels2-3 hours by road, by boat and on foot over harsh terrain to reach the centre from the closest gas station. This paper describes the software and hardware of a microgrid system that was designed and deployed at KBFSC to reduce the fuel consumption while improving duration of power availability. A key feature of the energy management software is a collaborative scheduler interface that provides visitors at the centre the choice of scheduling appliance usage. The system optimises generator active hours using a customised DG Optimiser technique, to ensure minimum diesel consumption. Simulations extrapolating from empirical data suggest that our system could reduce diesel consumption by a third, and total cost by $20 \%$, while making power available 24 hours a day. In addition, a user study with 8 visitors and 4 administrators showed that the collaborative scheduler interface is effective and usable.
\end{abstract}

\section{Categories and Subject Descriptors}

C.3 [Computer systems organization]: Special-purpose and application-based systems

\section{General Terms}

Design; Experimentation; Performance

\section{Keywords}

Microgrid; Scheduling; Dynamic Programming

\section{Introduction}

Microgrids [10] are emerging as a potential solution to the problem of remote electrification, where it is either im-

\footnotetext{
*Work completed at IBM Research

(C)2014 Association for Computing Machinery. ACM acknowledges that this contribution was authored or co-authored by an employee, contractor or affiliate of a national government. As such, the Government retains a nonexclusive, royalty-free right to publish or reproduce this article, or to allow others to do so, for Government purposes only.

BuildSys'14, November 5-6, 2014, Memphis, TN, USA.

Copyright 2014 ACM 978-1-4503-3144-9/14/11 ...\$15.00

http://dx.doi.org/10.1145/2674061.2674079
}

practical and/or uneconomical to extend the electricity grid [3]. Microgrids combine local sources of energy to form a miniature grid to which consumers can be connected to draw power as needed. While renewable sources of energy are picking up pace, a majority of remote locations still rely on diesel generators (DGs) [4] since they are simple to set up, lower in capital expenditure, and are available for use ondemand. The inherent remoteness of these locations makes transportation of diesel to these places an arduous task. The Kuala Belalong Field Studies Center (KBFSC) is a remote campus located within the scientific zone of a tropical rainforest in the Temburong District of Brunei Darussalam [8]. The center is not connected to the electricity grid, and is instead powered by a diesel generator. The generator at KBFSC is typically operated on a fixed schedule between $6 \mathrm{am}-8 \mathrm{am}$ and $4 \mathrm{pm}-11 \mathrm{pm}(\approx 9$ hours/day $)$, consuming 30-50 litres of diesel per day. The diesel is periodically supplied through a laborious process involving road- and water-based transport.

The objectives for an energy solution at KBFSC were threefold: (i) increase the duration of power availability, (ii) reduce diesel consumption, and (iii) minimize user inconvenience. Through discussions with administrators and visitors at the center and through energy instrumentation and analysis of power and fuel data, we discovered a number of issues. First, we found that during much of the fixed times that the generator was turned on, it powered only light loads. The DG was, therefore, frequently loaded to only $30 \%$ of its capacity (Fig. 1). Through further instrumentation, we discovered that the fuel efficiency characteristics of the DG is particularly non-linear. While efficiency non-linearity is inherent to all generators [18], DGs are typically sized to operate in the relatively flat portion of their efficiency curve. At KBFSC, however, the DG is sized for worst case load (a full center) and the average case load falls well below its flat portion (see Fig. 2). Improving this efficiency could lead us to significant diesel savings without affecting the overall energy generation.

A second aspect of the energy problem stemmed from fixed generator timings. On one hand, the DG stayed on even during times when no (or small) loads were needed. On the other hand, consumers would need to time-shift their consumption just to use some appliances. The generator could 
be turned on outside these hours as well but only for special needs. When the DG was turned on for individual use, not only would the efficiency be low, but unnecessary loads may also turn on inadvertently, leading to further waste. For example, a water heater may turn on, but the hot water may not be used soon and its heat would leak and be eventually lost. Arbitrarily increasing the number of generator run hours led to wastage and additional diesel use, while reducing it led to consumer inconvenience.

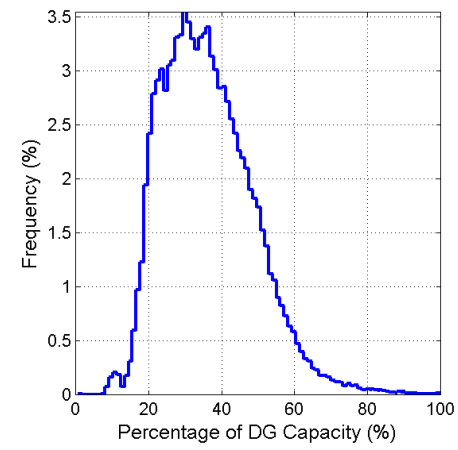

Figure 1: DG Generation Statistics - over 3 months

A dynamic system was needed that would meet user needs and improve systemic efficiency. A microgrid and its management system was designed for KBFSC to reach these goals. This paper describes the following three modules constituting the microgrid (Fig. 3):

- A battery bank is added to supply power to small but convenience (primary) loads, such as lights and fans. All other (secondary) loads, such as dryers, water heaters, etc., are powered only when the DG is on. The battery bank gets recharged when the DG is running. Sec. 4.1 provides details of the design of the battery sub-system.

- A dynamic scheduling system is developed to arrive at the best times and durations to run the DG. This is a collaborative scheduler (Sec. 4.2), which provides a user interface for visitors to choose when they want to use a particular secondary appliance. The scheduler uses an optimization system for each scheduling request to ensure minimum diesel consumption. Sec. 5.1 describes the user study that was performed to evaluate the interface.

- DG optimiser (Sec. 4.3) software uses characteristics of DG, load of secondary appliances, and current battery status, to suggest DG run hours. This results in reduction in fuel consumption as the DG is loaded optimally. Sec. 5.1 evaluates the efficacy of the optimization system.

Certain aspects were kept in mind while designing the system. First, the appliances used in KBFSC should not need individual energy monitoring capabilities. Second, the collaborative scheduler system should need minimal learning and minimize inconvenience caused to visitors as well as administrative staff. Third, the scheduler interface needs to be responsive, i.e., the DG optimiser should be low on compu- tation time. Our proposed system performs optimal scheduling of the DG based on visitor scheduling requests and battery charge status. We use simulations that extrapolate from real data to highlight the advantages of this solution strategy. We found that scheduling and optimizing the DG with visitor inputs using the collaborative scheduler results in $33.3 \%$ diesel fuel burn reduction and shaves total cost by $20.1 \%$ for 25 schedule requests, while making power available 24 hours a day for primary loads. In addition, we conducted cognitive walkthrough of the collaborative scheduler interface with 8 visitors and 4 administrators, to qualitatively evaluate the usability of the interface, and incorporated changes that were found during this user study. Our solution could be applied to other remote locations (such as rural communities) where community effort toward conservation can achieve higher energy efficiency and increased power availability for important loads.

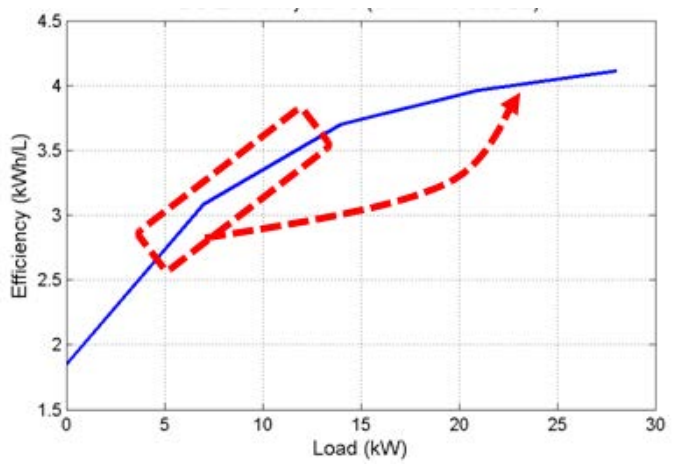

Figure 2: DG Efficiency Characteristics

\section{Related Work}

\subsection{Microgrids}

Microgrids are considered the modern, small-scale versions of the centralized electricity system [10]. They are ideal for remote locations, like the Maldives [16], and for rural electrification where connectivity to the macrogrid is infeasible. Microgrids enable local integration of renewable sources and could encourage conservative practices through community participation. Much research in microgrids has focused on their design and sizing. Hafez et al. [11] use the popular HOMER [2] tool to evaluate different microgrid designs, in terms of performance and sizing from an economic perspective. Microgrids are operationally challenging, however, due to the heterogeneous characteristics of sources and loads. In this paper, we focus on the optimal economic operation of the hardware components, using inputs from users through software interfaces and optimization algorithms.

\subsection{Interfaces for Sustainability}

Most of the sustainability related research works in interfaces have focused on designing eco-feedback technologies [9]. In particular for energy, researchers have used portallike interfaces to provide energy consumption information to the users. Using ways like goal setting, social comparisons, consumption visualization, and rewards, such systems have resulted in a decrease in consumption by $5-20 \%[13,9,7]$. 
However, all such systems are passive in nature, as they provide past consumption information to motivate consumers to reduce consumption in future. In contrast, our system is a pro-active system, asking consumers to plan their energy consumption a day in advance.

\subsection{Optimal Operation of Microgrids}

The problem of scheduling energy sources in a microgrid setting has been considered in prior literature. Morais et al. [15] use mixed-integer linear programming methods to solve the optimization formulation. This approach makes it difficult to include realistic constraints, and is also computationally intensive, thus requiring time. Other research work use genetic algorithms for computing optimal schedules [12], but randomized search algorithms have large memory requirements, slow computation times, and do not guarantee optimality. In this paper, we propose a two-step optimization procedure, which is a fast way of computing optimal schedules. It can easily incorporate scheduling constraints and non-linear system dynamics. Since operating norms at KBFSC preclude frequent rescheduling of the DG operating hours, knowledge-based expert systems such as those described in Ross et al. [19] are not relevant to this work.

\section{System Requirements}

We conducted semi-structured interviews with 4 visitors and 2 administrators. The aim of this study is to understand in detail the issues faced at KBFSC.

\subsection{Method}

All the interviews were conducted in the dining area of KBFSC, were voice recorded and later transcribed in English. The interviewer took extensive notes during the study. On an average, an interview took $\approx 1$ hour. Notes and transcripts were used for analysis. Transcriptions were open coded by one author. Two authors then jointly conducted selective coding to identify themes that were representative of the data and were either novel or important.

\subsection{Participants}

Four visitors ( 3 male, 1 female, average age $=30.5$ years, $\mathrm{sd}=2.6$; named V1:V4) participated in the study. Three of the participants have an educational background in Ecology, while one in Biology. All, except one, has visited a field center before. All the visitors answered in neutral to strongly agree (on a 5-point Likert scale, with 1 for strongly disagree and 5 for strongly agree) when asked about their orientation towards conservation, "I am environment-friendly and do everything possible to save the environment?" with average score of $4(\mathrm{sd}=0.8)$. With regards to DG run hours at KBFSC, all the visitors selected 'runs more than required' out of the four available options.

Two administrators ( 1 male, 1 female, average age $=43.5$ years, $\mathrm{sd}=0.7$; ; named A1:A2) participated in the study. Their education level varied with 1 having Bachelor's degree and 1 completed secondary school. With regards to their orientation towards conservation, both rated themselves high with score of 5 .

\subsection{Findings}

Visitors and administrators mentioned the following problems faced at KBFSC. (i) Fixed DG hours: A visitor and an administrator complained about limited electricity, "electricity is only available for certain fixed hours" (V1). On the contrary, other visitors mentioned that "DG run hours is generous" (V2, V3), and "DG is ON for more than required, especially in the morning as people usually sleep late or go out for field work." (V2, V4). This hints that the solution should aim to reduce the total number of DG run hours, by providing dynamic DG hours.

(ii) Underutilized DG: Underutilization of DG capacity was a problem identified by the administrators, as well as the visitors. From the KBFSC DG data, we found that the DG is most frequently loaded to only $30 \%$ of its capacity. At times, visitors noticed that the DG was running just for 1 visitor, "silly to run the DG just for one person" (V2). Administrators were looking for a solution that can use the DG at near-maximum capacity to obtain higher efficiency, " $D G$ should be exploited the maximum during the time it is running" (A2).

(iii) Inconvenient DG Hours: Each visitor wanted DG to run at different hours, as per their schedule and convenience. Two visitors mentioned taking hot shower in the morning before starting for field work, while others wanted to take hot showers in afternoon after they are back from the field visit. Visitors suggested that, "we should be involved in the decision-making process of the DG run hours, as we are the ones using it maximally... also, some of us might be unhappy with the current (DG) hours." (V2), “... tailor the DG hours as per the group who is here." (V4)

(iv) No DG = No Primary Loads: "No fans at night" (V2), "no lights at night... I use head lamps" (V3), and "no fans at day, when it gets hot" (V4), were some of the problems cited by the visitors due to unavailability of lights and fans (primary loads) during DG OFF hours. One of the solutions is to use batteries to power primary loads, when the DG is OFF.

During the stay at KBFSC, one of the authors observed two interesting phenomenon:

(i) Every night after dinner, all the visitors used to note their next day's activity schedule on a white board placed in the dining area, including time details, boats required, guide required, food required, etc. After all the visitors completed adding their details, the administrator would allocate boats and guide (by writing boat number, boat rower name, and/or guide name, on the white board) to each visitor/team for the time period.

(ii) Due to fixed DG run hours, visitors regularly plan their day in advance and shedule their activities (such as wake up early for hot shower, plan to do laundry, etc.) as per the DG run hours.

\section{System Design}

Based on the issues raised above, a solution was developed for the energy situation at KBFSC as illustrated in Fig. 3. A collaborative scheduler receives user inputs for their choice of secondary appliance usage. This is used by the DG optimiser to compute an optimal generator schedule. The optimiser uses DG efficiency characteristics and a model of the battery bank to ensure minimum diesel usage, while meet- 


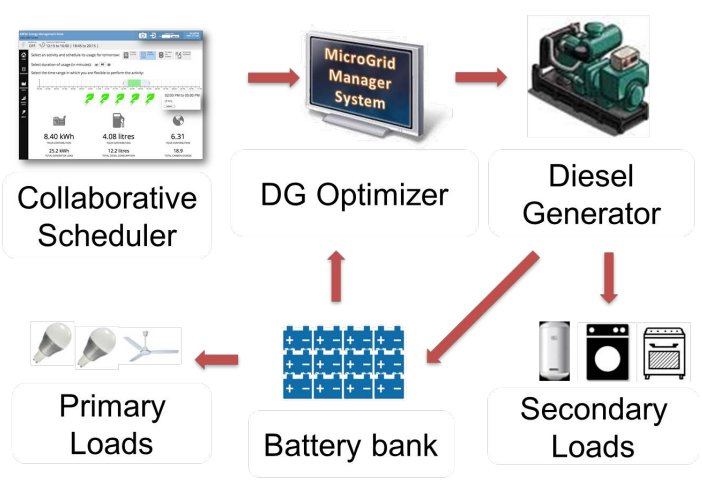

Figure 3: KBFSC Microgrid Solution Overview

ing user needs and maintaining sufficient battery charge to guarantee power availability for primary appliances. In this section, we discuss the design considerations for the battery bank, details of the collaborative scheduler interface for visitors and staff members to place scheduling requests, and interface for administrator to select the final DG run hours, followed by a description of the DG Optimiser.

\subsection{Battery and DG System}

The DG at KBFSC is a 30kVA three-phase system. With the DG being the sole source of power, user appliances can only be used during the fixed times it was on. Through interactions with users, we realized that a small amount of power, if it was available on demand all the time, would increase user convenience significantly. A storage based system seemed like a good choice. However, it would need to be connected to selected (primary) loads only to prevent inadvertent wastage or deliberate use of large appliances. A battery bank would need be installed, and would charge from DG power when it was running and provide power to loads when the DG was off.

Introduction of the battery bank could lead to another potential advantage. Fig. 2 illustrates the variation in fuel conversion efficiency of diesel generators with increasing load [1]. A higher loaded DG is typically more efficient in the amount of electrical energy it delivers per liter of fuel. The battery bank could play the role of load aggregation so that it would load the DG at a higher value and a higher efficiency while charging. The battery would then release this energy slowly over time to the low powered primary appliances when the DG is turned off.

For the system to be effective, energy losses that are part of the round trip to the battery and back though the inverter and charger would need to be offset by the efficiency gains of the DG. This implies that the battery bank should be charged at the maximum feasible rate, to load the DG as much as possible. Of course, the total rating of the DG (30kVA in our case) should never be violated. Lead acid batteries were chosen in our installation for their technological maturity and environmental stability. Lifetimes of batteries are sensitive to the charging and discharging rates they are subjected to [6]. This sensitivity is typically rated in terms of a fraction of their capacity. The final size of the battery was calculated based on a combination of the required charging rate (to be compatible with the size of the DG) and the capacity required for powering at least the primary loads for a duration of 24 hours.

\subsection{Collaborative Scheduler}

Collaborative Scheduler is a system that allows KBFSC visitors and staff members to schedule their appliance usage a day in advance, and helps administrators schedule DG run hours based on the inputs received from visitors and staff. The collaborative scheduler user interface is designed as a walk-up-and-use kiosk-based system with a minimal learning curve, and requires minimal interaction to complete the task. The interface was deployed on a touch-screen monitor connected to a (hidden) laptop. The monitor is placed in the dining area providing maximal visibility. There are two separate interfaces - Visitor and Administrator - which are discussed below.

\subsubsection{Visitor Interface}

The visitor interface (Fig. 4) allows visitors and staff members to schedule their appliance usage for the next day. A user (a visitor or a staff member) can walk up to the touchbased kiosk and select an activity that they would perform the next day, such as take a hot shower, wash clothes, dry clothes, etc. After an activity is selected, all the input fields (duration of the activity and time period to perform that activity) get auto-populated, so that the user can simply tap on 'Submit' and complete the task. After scheduling once, the user can return to the home screen to schedule another activity.

Advanced users can alter the different input fields to further customize their schedule. A user can increase or decrease the duration of an activity in steps of 15 minutes, using the ' + ' and '-' button (minimum: 15 minutes, maximum: 180 minutes). As per the selected activity and duration, the system uses DG Optimizer (Sec. 4.3) to recommend a time period to perform that activity (shown in green color, Fig. 4). By default, the selected time period (shown in grey color, Fig. 4) appears to be of the length of the selected duration and over the recommended time period. The user can drag the selected time period to choose another time period to schedule the activity. Moreover, the user can provide his/her time period flexibility of performing the activity, by choosing a time period bigger than the selected duration (e.g., in Fig. 4, selected duration is 60 minutes, while selected time period is 3 hours from $2-5 \mathrm{pm}$ ) using the handlebars provided on both sides of the selected time period. Based on the inputs, including activity, duration, and selected time period, the user is provided feedback in terms of greenness of the activity (using 5-leaf green level), and their activity's contribution towards the energy consumption, diesel usage, and carbon dioxide production, along with the total values for each. Greenness is inversely proportional to the selected duration, and directly proportional to the selected time period, and overlap between selected and recommended time period.

Flexibility and recommended time are directly related to conservation (or green) behavior, while duration is inversely related to the conservation behavior. To achieve a 5-leaf green level, the user needs to select a short duration for an ac- 


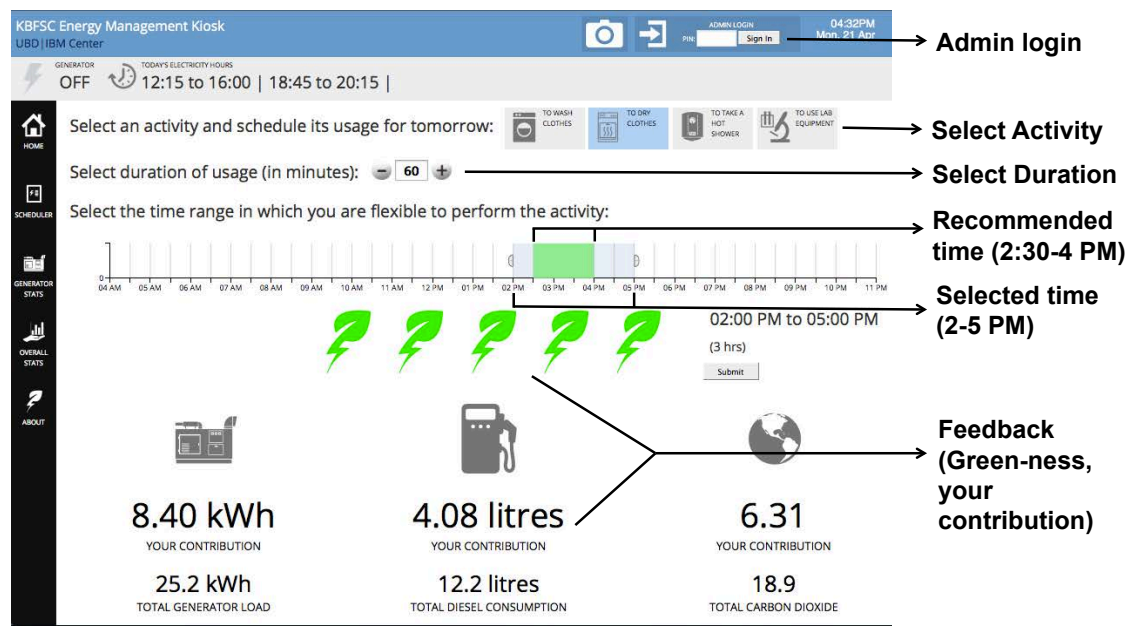

Figure 4: Visitor Interface of KBFSC Collaborative Scheduler

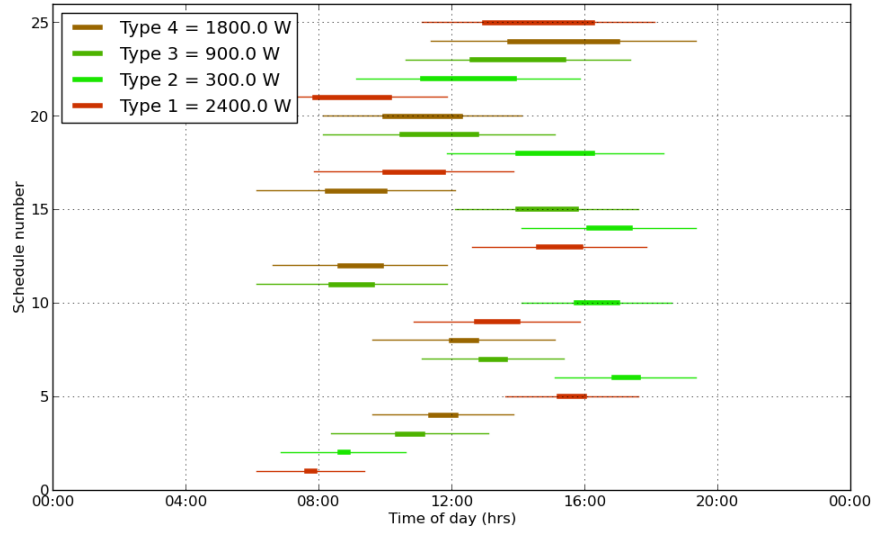

Figure 5: Schedules input by visitor with flexibility

tivity (e.g., 15-min hot shower, 60-min dry clothes, etcetera), and select a time that falls in the recommended time period and is highly flexible (for example, if a 60-min activity has a recommended time of 2:30-4 pm, the user should select something like $2-5 \mathrm{pm}$ ). Fig. 5 shows an example of inputs provided by several visitors. Thick lines indicate duration, while the thin lines indicate selected time period. The colors indicate appliances with different rated power.

\subsubsection{Administrator Interface}

The administrator interface (Fig. 6) allows viewing of activities scheduled by the visitors and staff members, to help the administrator schedule diesel generator (DG) running hours for the next day. To login, the administrator needs to tap on 'Admin Login' (Fig. 4), which pops up an on-screen numeric pad to enter the 6-digit administrator password. After entering the correct password, administrator interface is shown (Fig. 6). Based on the inputs received from the visitors and staff members, recommended DG timings gets autopopulated (shown as green star, Fig. 6) using DG Optimiser (Sec. 4.3), so that the administrator can tap on 'Publish' to complete the task.

In addition, the administrator can view all the scheduling requests and is free to select any DG run timings (irrespective of the DG Optimiser's recommendations). The top bar with green regions visualises the currently selected DG run timings, which are also being listed on the right hand side. The bar below the selected DG run timings visualisation is the activity bar. The activity bar is interactive and has yellow regions visualising the time periods, during which users have scheduled any activity. Administrator can use the handlebars to select a time period on the activity bar. Appliances being scheduled during the selected time period get populated in the pop-up below it, and the two graphs visualising power consumption and diesel usage gets automatically updated, along with the aggregate data and contribution of the current time period selection on the right. Tapping on the 'Allot' button adds that time period to selected DG run timings.

\subsection{DG Optimiser}

The optimal times of operation of the diesel generator are computed based on the scheduling requests received from the scheduler interface for the secondary appliances, and current battery charge level with battery charging/discharging chemistry. The scheduling requests include the power rating of the appliance for the requested activity, the usage duration, and the acceptable times of operation (i.e, selected time period). The objective of the optimisation procedure is to minimize the diesel consumption. There are two facets to the problem formulation for this system. First, there is the problem of scheduling the actual running time of each request. Second, there is the problem of computing the DG running schedule.

Ideally, the globally optimal solution can be computed by optimizing the appliance and DG running times together in one step. However, this methodology faces severe computational issues because of the size of the state space. For illustration, let us assume that the optimisation time window is divided into 96 slots of 15 minutes each, covering a total of 24 hours. Let the number of scheduling requests be 25 . The number of combinations of appliance running times is of the order of $96^{25}$. This will be further multiplied by the number of combinations of DG running times, thus making the computation highly time-consuming. Instead, we use a two-step 


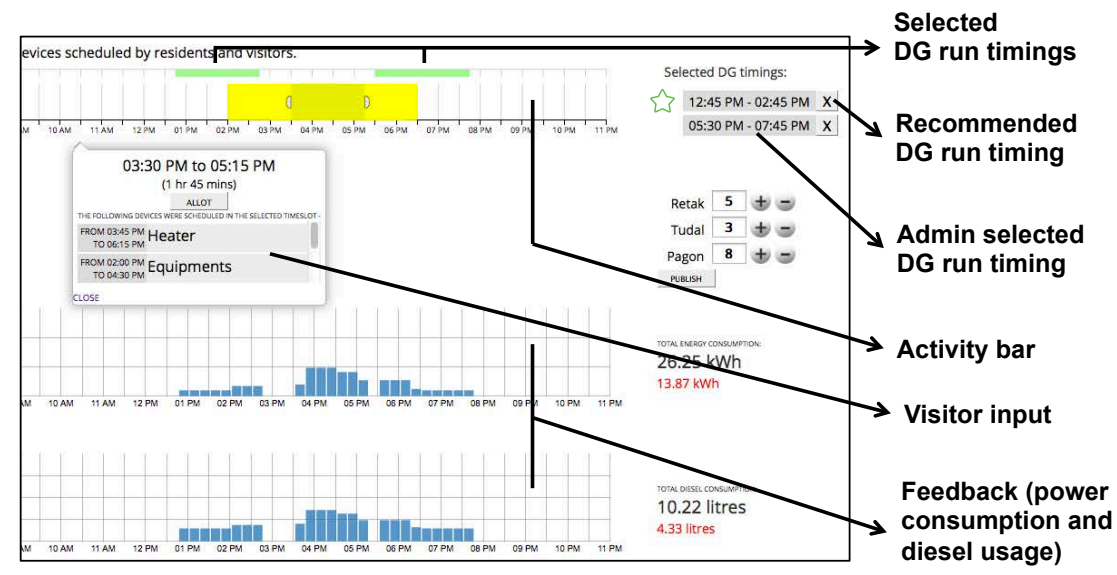

Figure 6: Administrator Interface of Collaborative Scheduler

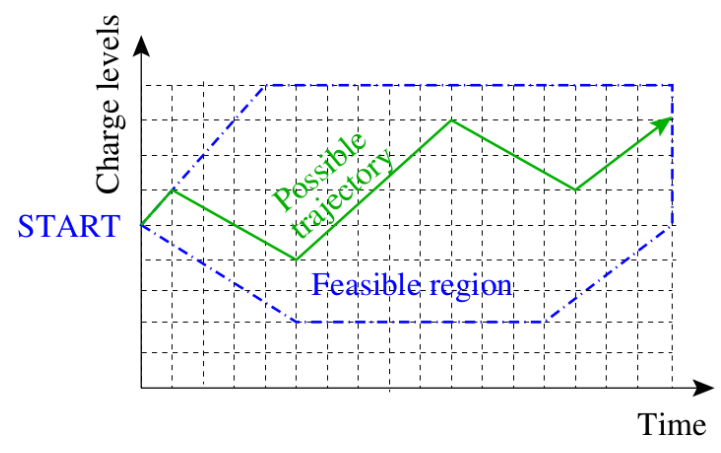

Figure 7: Feasible state space representation of DG Optimiser

optimisation procedure that schedules the appliance running time according to a heuristic, and then computes the optimal DG running times using a dynamic programming approach. Due to the complex dynamics of the battery and the shape of the diesel efficiency curve, the optimisation problem for the second step is non-convex and non-linear. Combined with the set of constraints, dynamic programming was considered to be the simplest option for obtaining the optimal solution.

The solution to the first step is motivated by the shape of the generator efficiency curve shown in Fig. 2. Note that the efficiency is highest when the generator is loaded close to its capacity. Additionally, the total energy consumption is constant, regardless of the optimisation procedure. Therefore, we introduce a heuristic that aims to synchronize as many appliances as possible, at any given time. This allows the generator to shut down and save diesel in the gaps in between appliance demand times. The algorithm assigns appliance running times one at a time, starting with the most constrained appliance (i.e with minimal padding between usage duration and selected time period). Successive appliances are then scheduled by maximizing the overlap with already scheduled appliances. After one pass through the list of requests, a schedule of all secondary loads and their aggregate power profile is generated. This schedule is then conveyed to the second step of the optimisation procedure.

In the second step, we schedule the DG using a dynamic programming formulation. The load power profile has al- ready been defined by the first step. This profile, in addition to the primary loads, defines the constraints of the problem. The scheduling of the generator may be subject to one further constraint, depending on the circuitry for powering secondary loads. In the simplest case, both primary and secondary loads run from the battery, and the generator is used only to recharge the battery. In this case, no additional constraint is imposed on the generator schedule. However, this architecture implies a higher wear and tear of the battery. It may also result in a demand profile that exceeds the current capacity of the battery. Moreover, as electricity is freely available from the battery at any time of the day, users may tend to be less economical in their appliance usage. That's why we implemented an architecture where all secondary loads are run directly from the DG, and the battery only powers smaller primary loads. The resulting design is incorporated into the mathematical formulation by constraining the generator to be $\mathrm{ON}$ whenever one or more secondary appliances are scheduled to run, according to the first step of the optimisation. In Sec. 5, we show that the resulting deterioration in the optimal fuel cost is minor, compared to the gains in battery life and the encouragement to users to be economical in their appliance usage.

The system state and dynamics for the battery bank employs the KiBaM model for lead acid batteries [14]. In the KiBaM model, the battery is modelled as two interconnected charge wells, consisting of available charge and bound charge. The state of each charge well is discretized into $M$ intervals, giving $M^{2}$ possible charge state combinations. The time interval of optimisation is discretized into $N$ intervals. The cost in each interval is the amount of diesel consumed by the generator, which is 0 if the generator is $\mathrm{OFF}$, and is greater than 0 otherwise. In order to incorporate the spool-up fuel consumption of the DG, a constant term $F_{\text {start }}$ is added to the stage cost each time the DG is switched $\mathrm{ON}$ from an OFF state. Let $i$ denote the time index of a time slot of duration $\tau$, say 15 minutes. Let $c(i)$ be the state of charge of the battery at time $i$, and $P_{B}(c(i))$ be the average power received by the battery if the DG was running for $\tau$ starting from the battery state $c(i)$. Let $F_{B}(c(i))$ be the 
corresponding fuel consumption for the diesel generator, as calculated from the relevant DG consumption data sheet [1]. Note that the energy received by the battery is a function of its state of charge and dynamics in the battery model [14], and hence drives the fuel consumption of the generator. As explained previously, the battery lifetime depreciation is included in the system model [6]. The objective function for the optimisation procedure is,

$$
J=\sum_{1}^{N} u_{i}\left[F_{B}(c(i))+F_{S}(i)+\left(1-u_{i-1}\right) F_{\text {start }}\right] .
$$

Here, $u_{i} \in\{0,1\}$ is the binary decision taken at time $i$, controlling the operation or otherwise of the DG. $P_{S}(i)$ is the expected power profile of secondary appliances in time step $i$ derived from the first optimisation step, and $F_{S}(i)$ is the corresponding diesel usage. The spool-up cost $F_{\text {start }}$ is counted only if the DG is running in the current time period, and was not running in the previous time period. It is assumed that its initial state is $u_{0}=0$. The optimal schedule can be computed by using Dijkstra's algorithm to solve a shortest path problem [5] from the initial state $(i=0$ and battery charge equal to the initial charge level) to each feasible final state $(i=N$ and the battery's final state of charge lower bounded by its initial state of charge). The optimisation algorithm begins from a known initial state at $i=0$. It explores each of two options: battery charging (generator $\mathrm{ON}$ ) or battery discharging (generator OFF), and arrives at two potential destination states accordingly. The cost to reach each state is the diesel consumed by the sequence of decisions culminating in the current state of charge at the current time. This cost is saved by the algorithm, along with the battery state in the previous time step and the decision taken at the previous time step. The algorithm proceeds in this way to the end of the time window, where it is constrained to only consider final states that replenish the battery levels to their starting values, or higher.

The optimal control computation algorithm is given in Algorithm 1. We define the following matrices of size $M^{2} \times(N+1)$, with rows representing state of charge of the two batteries and columns representing time. Note that the $(N+1)^{\text {th }}$ column represents the end of the final time period.

- $\mathcal{F}$, with each element containing the minimum fuel to reach state $(j, i)$ where $i$ is an integer, $0 \leq i \leq N$,

- $\mathcal{C}$, with each element containing the charge level at time step $(i-1)$ on the optimal path from $(c(0), 0)$ to $(j, i)$, and

- $\mathcal{D}$, containing the decision taken at time step $(i-1)$ on the optimal path from $(c(0), 0)$ to $(j, i)$.

This procedure is illustrated schematically in Fig. 7, with time on the $\mathrm{X}$-axis, and potential combinations of charge levels on the Y-axis. The starting state is well defined, while the acceptable final charge levels form a subset of all the potential charge levels. By accounting for battery dynamics, a feasible region such as the one depicted in Fig. 7 is automatically defined and explored by the dynamic programming algorithm. If the level of each charge well in the KiBaM [14] battery model is divided into $M$ intervals, the maximum number of reachable states at any time step is $M^{2}$,

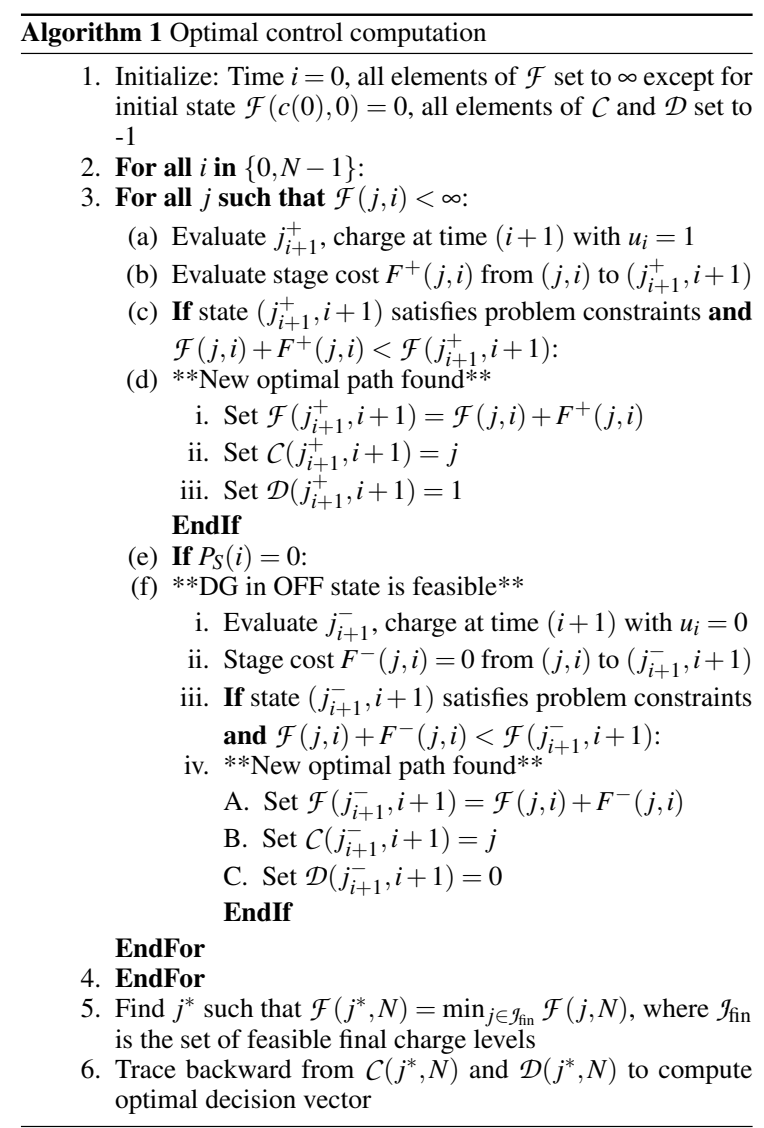

and the number of decisions available at any state of charge is at most 2. Thus, the maximum number of computations required to find the optimum generator schedule is $2 N M^{2}$. The computational complexity thus scales linearly with the time dimension.

\section{Results}

\subsection{DG Optimizer Evaluation}

In this section, we estimate the benefits delivered by the optimisation procedure using simulations that extrapolate from empirical data. DG and battery model (KiBaM) parameters used in this section were taken from vendor data sheets. It is assumed that users use the interface described

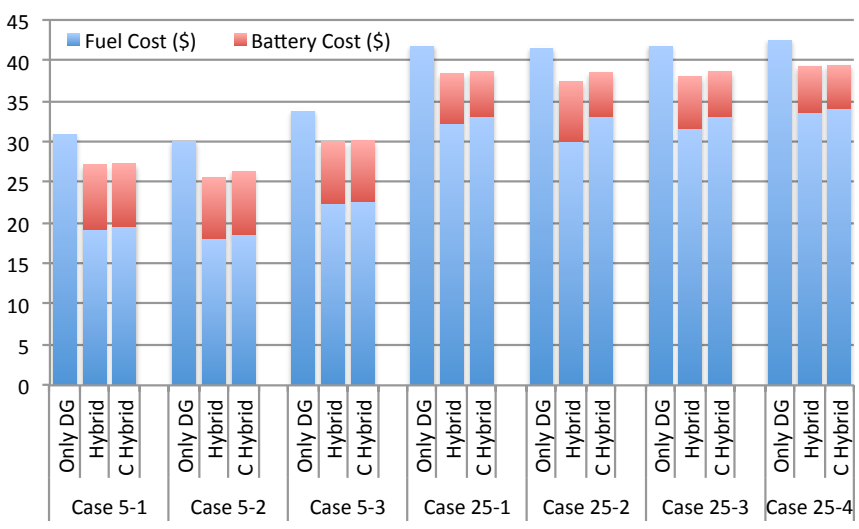

Figure 8: Cost comparison between Only DG, Hybrid \& C-Hybrid 

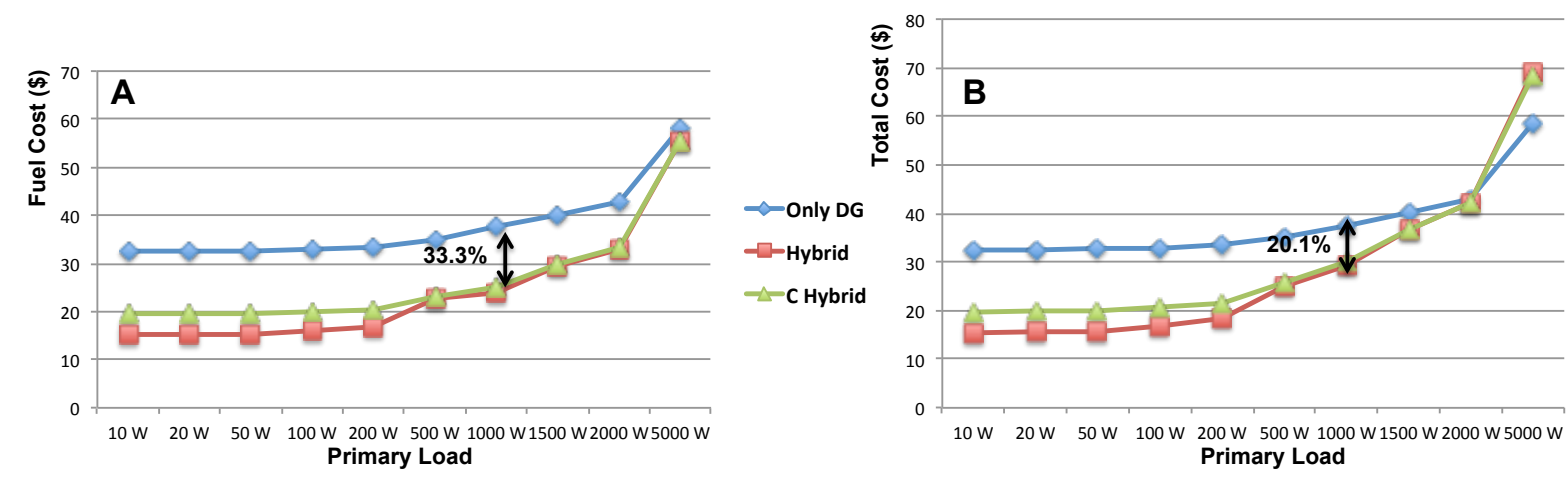

Figure 9: Cost sensitivity (a. Fuel cost, and b. Total cost) to variation in primary load

in Sec. 4.2 to communicate their secondary appliance usage preferences. Each scheduling request includes the power rating of the appliance, the expected duration of usage, and the acceptable time window of operation (i.e, selected time period). A sample set of inputs is shown in Fig. 5, which depicts 25 scheduling requests over the course of a day. The earliest feasible time for any request is 6:00 am, and the latest feasible time is $7: 30 \mathrm{pm}$. Several such inputs were generated for analysis: 4 input cases with 25 schedule requests each (Case 25-1 to 25-4), and 3 input cases with 5 schedule requests each (Case 5-1 to 5-3). The inputs were based on an analysis of measurements taken for ten appliances at KBFSC over three months.

Based on the description in Sec. 4.3, we compared the costs associated with three possible architectures. First, to run the generator whenever there is non-zero demand (without any optimisation, which is how KBFSC currently operates). This option is referred as 'Only DG' in Fig. 8. Second, it is possible to run all appliances from the battery, with the generator being used only to optimally recharge the battery. This option is referred as 'Hybrid'. Finally, the third architecture runs all primary appliances from the battery, and all secondary appliances directly from the generator. This option is referred as 'C-Hybrid', which implies a hybrid architecture constrained to operate the DG for all secondary appliances. The resulting total costs are compared in Fig. 8 for all seven input cases. The total cost consists of the cost of diesel used over the course of the day, as well as the amortized depreciation in battery life because of its usage. Even after including the cost of the battery, it can be seen that there is a significant cost reduction in moving from the former architecture to one of the two latter ones. While the C-Hybrid case does not perform as well as the pure Hybrid case, it can be noted that the increase in cost is very small. When taken in context of the battery capacity costs and human factors described in Sec. 4.3, this is likely to be an acceptable tradeoff.

The sensitivity of the optimal cost to the magnitude of the primary load is depicted in Fig. 9. The value on the X-axis is the mean primary load throughout the course of the day. It is seen that the two hybrid architectures always have lower diesel fuel cost when compared to the generator-only architecture. Note that the constrained hybrid case has higher fuel cost than the pure hybrid case when the primary loads are small (Fig. 9A). This is because of the constraint that the generator must run whenever secondary loads are operating. When this constraint is removed for the pure hybrid case, the generator can push sufficient charge into the battery to supply both primary and secondary loads, while still running for a shorter duration of time. If we include the cost due to a decrease in the battery life, the total cost for the hybrid architectures is higher than that for the generator-only architecture, for large primary loads (Fig. 9B). The parity point occurs at $2 \mathrm{~kW}$. For primary loads higher than this value, the decrease in battery life is too rapid to be offset by the reduction in the cost of diesel. At the expected primary load level at KBFSC $(1 \mathrm{~kW})$, the expected cost savings are $33 \%$ for fuel only, and $20.1 \%$ when the battery cost is included.

Finally, we consider the effect of incorporating the cost of spooling up the generator (change from OFF to ON state). In Fig. 10, we demonstrate the effect for the input case shown in Fig. 5. The $\mathrm{X}$-axis denotes the time of day, while the $\mathrm{Y}$-axis depicts the generator state $(\mathrm{OFF} / \mathrm{ON})$ in each 15 -minute time period. If the cost of starting the generator is ignored, the optimisation procedure would produce a generator schedule with 11 OFF to ON switches (blue circles in Fig. 10). When a cost of 2 USD is assigned to each switch event, the optimiser instead produces a schedule that contains only 2 OFF to ON switches (black stars in Fig. 10). We do not compare the fuel cost for the two cases described here, because ignoring the spooling cost of switching $\mathrm{ON}$ the generator is not a realistic assumption.

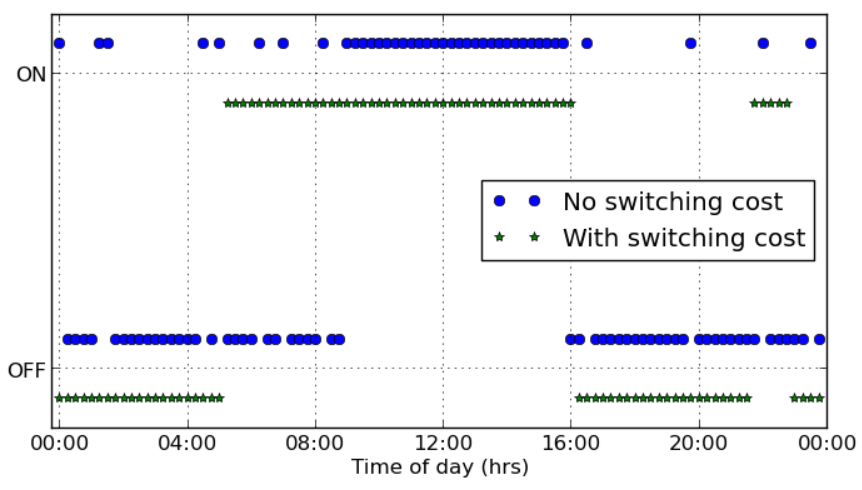

Figure 10: DG cost analysis with and without switching cost 


\subsection{Collaborative Interface Evaluation}

We conducted cognitive walkthrough with 8 visitors and 4 administrators. The aim of this study is to evaluate the usability of both the visitor and administrator collaborative scheduler interface. The interface presented in Sec. 4.2 is the final version that was deployed, which incorporates several changes that were found through the cognitive walkthrough evaluation.

\subsubsection{Method}

As both the visitors and administrators have to perform specific tasks - scheduling appliances usage and scheduling DG run hours, respectively - we used a task-specific qualitative evaluation method, Cognitive Walkthrough [20]. The participants were asked to think aloud through out the study. Visitors were asked to schedule their appliance usage for the next day in basic mode with minimal input (only activity selection and 'submit'), and in advanced mode with maximal customization (including activity selection, duration selection, time period selection, and 'submit'). Similarly, administrators were asked to login, schedule DG run hours for the next day, with minimal inputs and with maximal customization.

Similar to the interviews, all the walkthroughs were conducted in the dining area of KBFSC, audio-recorded and later transcribed in English. Rest of the analysis details for walkthroughs is the same as semi-structured interviews (Sec. 3.1).

\subsubsection{Participants}

Eight visitors ( 4 male, 4 female, average age $=29.1$ years, $\mathrm{sd}=11.7)$ participated in the study. The visitors were from different locations - 5 from USA, 2 from Brunei and 1 from Indonesia. All the visitors have an educational background in Ecology; six of the visitors were PhD students, one was a Research Assistant, and one was a Professor. All of them were visiting KBFSC for a 10-days long field study Ecology course; four of the visitors have visited a field study center before. All were aware of their monthly electricity bills and per unit energy pricing at their residence. All the visitors answered in neutral to strongly agree (on a 5-point Likert scale) when asked about their orientation towards conservation, with average score of $3.8(\mathrm{sd}=0.6)$. With regards to DG run hours at KBFSC, all (except one, who selected 'runs for optimal hours') the visitors selected 'runs more than required'. In addition, all the visitors were Internet savvy, with an average self-score of 4.2 ( $\mathrm{sd}=0.8)$, on a 5-point Likert scale, with 1 lowest and 5 highest in terms of Internet usage.

Four administrators ( 2 male, 2 female, average age $=41.5$ years, $\mathrm{sd}=5.1$ ) participated in the study. Their education level varied with 2 having Bachelor's degree, 1 having Master's degree and 1 completed secondary school. With regards to their orientation towards conservation, all rated themselves high with average score of $4.5(\mathrm{sd}=0.5)$. All, except one, were Internet savvy. (Note: Two of these administrators even participated in the semi-strcutured interviews.)

\subsubsection{Visitor Interface Findings}

Below are the key findings from the visitor collaborative schedule interface: (i) Addition of 5-leaf Green Level: In the initial design, based on the activity, duration, and time period selection, users were provided feedback only through their activity's contribution towards the energy consumption, diesel usage, and carbon dioxide production, along with the total values for each (lower portion of Fig. 4). During the cognitive walkthrough, we found participants struggling to understand the impact of changing activity, duration, or time period selection, as the feedback numbers kept changing according to any change in selection. It was hard for the participants to keep track of the three feedback numbers, to identify the best schedule selection. In addition, there was no goal to achieve, i.e., the upper limit of the "goodness of selection" (V5) was not clear. Hence we decided to add a new feedback parameter, 'greenness' of the activity (using 5-leaf green level, Fig. 4). This allowed the visitors to try and achieve maximum number of green leaves during their scheduling selection.

(ii) Duration Selection: In the intial design, duration selection used to be a text box with the number of minutes written inside it. On tapping it, a pop-up opened up to select any number of minutes. With the cognitive walkthrough, we found that participants were having difficulties in changing the duration, "what to click? should I click the box?" (V4). Moreover, visitors mostly chose period of 15-mins interval (such as 15 mins for shower, 30 mins for washing machine, 45 mins for dryer). Hence we decided to replace the number selector pop-up with the ' + ' and '-' button, allowing users to quickly increase or decrease the duration of an activity in steps of 15 mins (minimum: 15 minutes, maximum: 180 minutes).

(iii) Selected Time Period Animation: Using the handlebars provided on both sides of the selected time period, a user can provide his/her time period flexibility of performing the activity. The participants complained that, "though I selected $2 \mathrm{pm}$ to $5 \mathrm{pm}$, which is also showed on the text below, the (grey-colored) selection actually shows 2:10ish to 4:55ish" (V7). In the intial design, the selected time period window used to show the visitor's selection, and the text below used to round it up to the nearest time window, thus a 2:05-4:55 pm or 1:55-5:05 pm selection was written as 2-5 $\mathrm{pm}$. In the updated interface, as soon as the handlebars were released, the selected time period automatically expand, reduce, and/or transition to the nearest full time period window, such that the text and actual (grey-colored) selection were consistent.

\subsubsection{Administrator Interface Findings}

Below are the key findings from the administrator collaborative schedule interface:

(i) Selected DG Run Timings Visualization: In the initial version, the top bar showing DG run timings was not present. The selected DG timings were only shown as numbers on the top right (Fig. 6). During the cognitive walkthrough, we found that administrators require multiple attempts to select a non-overlapping time period on the activity bar using handlebars. As A3 pointed, "it is hard to read the time here (on the top right), while making the selection here (in the activity bar)." In the revised interface, we added a selected DG run timings visualization at the top of the activity bar, thus guiding the user in selecting non-overlapping period. 
(ii) Recommended DG Run Timing Star: Administrators showed interest in keeping the recommended DG timings, stating that "the system must have made intelligent decisions" (A2). However, after allotting multiple other DG run timings, the participants were not able to distinguish between the recommended versus self-selected DG run timings. Hence we added a green star to show the recommended DG run timings. Moreover, removing an administrator selected DG run timing is a 1-step process (clicking on the ' $\mathrm{X}$ ' button next to the text), while removing a recommended $\mathrm{DG}$ run timing is a 2-step process with a comfirmation pop-up as the second step.

\section{Discussion}

The system and methodology described in this paper does not explicitly account for schedule disruptions, which can be caused by a discrepancies between user input and actual usage. It is difficult to model the stochasticity introduced by this aspect, because the type and magnitude of discrepancy differs from one user to another. A direct method to account for stochasticity would be to implement a robust optimisation framework. This would involve replacing the minimisation in (1) with a minimax formulation [17], where the worst case (maximum) fuel cost is minimised. It is expected that the consequent modification required to the dynamic programming algorithm would be minimal. However, formal implementation of this methodology and its evaluation are a part of future work.

In this work, we demonstrated the value of implementing collaborative optimisation techniques for energy resources in a microgrid setting. However, the same methodology is potentially applicable to a wide spectrum of instances in the energy space. The core idea of the design effort was on providing feedback to consumers about the impact of their own activities on collective consumption. In addition, the system also provided suggestions about optimal scheduling of user demand. Implicit in this design is the coordination of energy consumption across users who may or may not be able to interact directly with each other. As such, the collaborative interface idea can even be applied to systems where the users are not collocated. Some examples of minimising ecological impact through coordination of activities can include, (i) Optimal scheduling of commercial shuttle services by having users $\log$ their planned times of travel to/from work for the next day, and, (ii)Planning of thermal storage (for example, pre-cooling) in office premises by using forecasts of building occupancy.

\section{Conclusions}

This paper describes the solution designed for reducing diesel consumption at KBFSC, a remote ecological field study centre in Brunei. The system employed a battery bank to increase power availability to primary loads and a collaborative scheduler for access to power for secondary loads. An optimiser ensures that the DG run at the appropriate times to minimize fuel consumption while keeping the batteries charged and meeting user needs. Simulations modelled on real data suggest that our system could reduce diesel consumption by up to $33.3 \%$ and total cost by $20.1 \%$. Moreover, our proposed system can provide power to the centre uninterruptedly, as oppose to only $\approx 9$ hours available in the past.

\section{Acknowledgements}

We would like to thank Pg Dr Iskandar Petra and Abdul Aziz bin Haji Mohamad Ali of the UBD|IBM Centre; Teddy Chua Wee Li, Muhammad Salleh bin Abdullah Bat and Hjh Masnah Hj Mirasan at KBFSC and Institute for Biodiversity $\&$ Environmental Research, UBD; and RadioStudio, for their support in this project.

\section{References}

[1] Cummins Power DG datasheets. http://www.cumminspower.com/.

[2] HOMER - hybrid optimization of multiple energy resources. http://www.homerenergy.com/.

[3] Solarising India: Commercial feasibility analysis and an implementation roadmap. http://www.ima-india.com/solarising-rural-india.pdf, 2006.

[4] The Rising Sun: Grid Parity Gets Closer. KPMG, 2012.

[5] Bertsekas, D. Dynamic Programming and Optimal Control, 2nd ed. Athena Scientific, 2000.

[6] Bindner, H., Cronin, T., Lundsager, P., Manwell, J. F., Abdulwahid, U., and Baring-Gould, I. Lifetime modelling of lead acid batteries. 2005.

[7] Erickson, T., Li, M., Kim, Y., Deshpande, A., Sahu, S., Chao, T., Sukaviriya, P., and Naphade, M. The Dubuque Electricity Portal: Evaluation of a city-scale residential electricity consumption feedback system. In SIGCHI Conference on Human Factors in Computing Systems (Paris, France, 2013).

[8] Faudzulani, A., Tennakoon, K., and Malai, A. Life Underneath the Canopy: The Kuala Belalong Field Studies Centre Showcase. Universiti Brunei Darussalam, 2011.

[9] Froehlich, J., Findlater, L., and Landay, J. The design of eco-feedback technology. In SIGCHI Conference on Human Factors in Computing Systems (Atlanta, GA, 2010).

[10] Galvin, R., Yeager, K., and Stuller, J. Perfect Power. McGraw Hill, 2009.

[11] Hafez, O., and Bhattacharya, K. Optimal planning and design of a renewable energy based supply system for microgrids. Renewable Energy 45 (2012), 7-15.

[12] Hong, Y., and Li, C. Short-term real-power scheduling considering fuzzy factors in an autonomous system using genetic algorithms. Proceedings of Generation, Transmission and Distribution 153, 6 (Nov 2006), 684-692.

[13] Kjeldskov, J., Skov, M., Paay, J., and Pathmanathan, R. Using mobile phones to support sustainability: A field study of residential electricity consumption. In SIGCHI Conference on Human Factors in Computing Systems (Austin, TX, 2012).

[14] Manwell, J., and McGowan, J. Lead acid battery storage model for hybrid energy systems. Solar Energy 50, 5 (1993), 399-405.

[15] Morais, H., Kadar, P., Faria, P., Vale, Z., and Khodr, H. Optimal scheduling of a renewable micro-grid in an isolated load area using mixed-integer linear programming. Renewable Energy 35, 1 (2010), 151-156.

[16] Nayar, C., Tang, M., and Suponthana, W. Wind/PV/diesel micro grid system implemented in remote islands in the republic of Maldives. In International Conference on Sustainable Energy Technologies (Nov 2008), 1076-1080.

[17] Osborne, M., and Rubinstein, A. A Course in Game Theory. MIT Press, Cambridge, MA, 1994.

[18] Prasetiawan, E. Modeling, simulation and control of an earthmoving vehicle powertrain simulator. $\mathrm{PhD}$ thesis, 2001.

[19] Ross, M., Hidalgo, R., Abbey, C., and Joos, G. Energy storage system scheduling for an isolated microgrid. Renewable Power Generation 5 , 2 (Mar 2011), 117-123.

[20] Wharton, C., Rieman, J., Lewis, C., and Polson, P. Usability inspection methods. John Wiley \& Sons, Inc., New York, NY, USA, 1994, ch. The Cognitive Walkthrough Method: A Practitioner's Guide, 105140 . 